\title{
Condição corporal e desempenho produtivo de cabras Alpinas no início de lactação
}

\author{
Larissa Pires Barbosa ${ }^{1^{*}}$, Marcelo Teixeira Rodrigues ${ }^{2}$, José Domingos Guimarães ${ }^{2}$, Vitor \\ Valério Maffili ${ }^{3}$, Lincoln da Silva Amorim ${ }^{4}$, Américo Fróes Garcez Neto ${ }^{5}$
}

\author{
${ }^{1}$ Programa de Pós-graduação em Zootecnia - Universidade Federal de Viçosa - UFV \\ 2 Universidade Federal de Viçosa - UFV. \\ ${ }^{3}$ Centro de Pesquisa Gonçalo Moniz - FIOCRUZ. \\ ${ }^{4}$ Faculdade Pio Décimo. \\ ${ }^{5}$ Universidade Federal do Vale do São Franscisco - UNIVASF.
}

RESUMO - Objetivou-se avaliar a influência da condição corporal ao parto sobre os parâmetros produtivos de cabras Alpinas no início da lactação. Sessenta e oito cabras foram distribuídas em três grupos, de acordo com o escore de condição corporal (baixo, entre 1,00 e 2,75; intermediário, entre 2,75 e 3,50; e alto, entre 3,50 e 5,00) e avaliadas do parto até a oitava semana de lactação. Realizaram-se o controle diário do consumo alimentar e da produção leiteira e avaliações semanais de peso corporal, escore da condição corporal e composição do leite. Os animais com escore corporal alto apresentaram menor consumo de matéria seca, fibra em detergente neutro e proteína bruta em comparação àqueles com escores corporais baixo e intermediário. O período médio de recuperação do consumo de matéria seca dos animais das três condições corporais foi de 16 dias (2,3 semanas) do pós-parto, com estabilização até o final do experimento. Na sexta semana de lactação, o peso corporal dos animais com escore corporal baixo se igualou ao daqueles com escore corporal intermediário. Não houve efeito da condição corporal ao parto nas variações de ECC e da produção leiteira ao longo do período. O teor de gordura do leite dos animais com escore corporal alto foi menor nas duas primeiras semanas pós-parto em comparação ao dos animais com escores corporais baixo e intermediário. Elevada condição corporal ao parto influencia negativamente o consumo voluntário de cabras leiteiras no início da lactação, mas quando se utiliza dieta de alta qualidade e com altos níveis de energia, a influência da condição corporal ao parto inexiste para variações de peso, de escore da condição corporal e produção leiteira dos animais.

Palavras-chave: consumo, pós-parto, produção leiteira

\section{Body condition and productive performance of Alpine goat in early lactation}

ABSTRACT - The objective of this study was to evaluate the influence of body condition at birth on Alpine goat productive parameters in early lactation. Sixty-eight females were assigned to three groups according to the body condition score (BCS) - low $(1.00-2.75)$; intermediate $(2.75$ - 3.50) and high $(3.50-5.00)$ and evaluated from kidding to the eighth week of lactation. The feed intake and milk production were recorded on a daily basis and the body weight, body condition score and milk composition at weekly intervals. Animals with a high body condition score presented lower dry matter intake, neutral detergent fiber and crude protein compared to those with low and intermediate body condition scores. The average time for dry matter intake recovery of the animals with the three body condition scores was 16 days (2.3 weeks) postpartum, and stabilization by the end of the experiment. In the sixth week of lactation, animals with low body condition score had the same body weight of animals with intermediate body condition score. There was no effect of the body condition at birth on the body condition score changes and milk production during the experiment. The milk fat content of animals with high body condition score was lower in the first two weeks postpartum compared to animals with low and intermediate body condition score. The high body condition at birth influences negatively the ad libtum intake of dairy goats in the early lactation, but when it is used feed of high quality with high energy levels, the influence of the body condition at birth is nil to variations in weight, BCS and milk production.

Key Words: intake, milk production, postpartum

Este artigo foi recebido em 3/8/2007 e aprovado em 27/11/2008. 


\section{Introdução}

A caprinocultura tem grande potencial como atividade econômica. A versatilidade dos caprinos em reproduzir-se nas mais variadas condições faz com que regiões mais favorecidas, do ponto de vista climático, intensificassem sua criação, visando atender à crescente demanda do consumo de seus produtos. A criação nestas regiõesvisa principalmente à produção de leite e derivados. Assim, é necessária a obtenção de animais de alta produção leiteira e de técnicas específicas que atendam essas necessidades de produção.

Com o aumento da utilização de animais leiteiros de alta produção, cresce a preocupação com o período pós-parto, por ser um período de grandes mudanças fisiológicas para as fêmeas e por refletir em grandes perdas econômicas.

Portanto, é importante, principalmente em animais leiteiros de alta produtividade, parirem com adequada reserva corporal para mobilização e atendimento aos requerimentos de energia e proteína durante o início da lactação (Rennó et al., 2006). Essa reserva pode ser subjetivamente avaliada pelo escore da condição corporal (ECC), que representa o acúmulo de gordura no tecido animal e tem sido uma metodologia prática no manejo de animais leiteiros.

Maiores informações são necessárias para determinar o ECC ideal ao parto em cabras leiteiras e nos vários estágios da lactação. Assim, este estudo foi realizado com o objetivo de avaliar as relações entre o ECC em cabras leiteiras ao parto e a resposta animal por meio da produção de leite e do consumo voluntário.

\section{Material e Métodos}

O estudo foi realizado no Setor de Caprinocultura do Departamento de Zootecnia da Universidade Federal de Viçosa (UFV), no Município de Viçosa, Minas Gerais. Foram utilizadas 68 cabras da raça Alpina, previamente selecionadas quanto ao escore da condição corporal, à ordem de parição (fêmeas de segunda e terceira lactações) e à produção leiteira, obtendo-se um grupo homogêneo e sem alteração clínica e reprodutiva.

Os animais foram confinados em baias individuais de piso ripado e com dimensões de 1,5 × 2,0 $\mathrm{m}$. Todas as cabras receberam a mesma dieta, composta de silagem de milho, farelo de soja (Glycine max L.), fubá de milho (Zea mays L.), calcário calcítico e sal mineral (Tabela 1), na forma de mistura completa, fornecida duas vezes ao dia (7h30 e $17 \mathrm{~h}$ ). A ração foi calculada para atender as exigências nutricionais de cabras em lactação com produção até 5 kg de leite/dia, de acordo com recomendações do AFRC (1997). Para que se permitisse consumo voluntário, uma quantidade adicional de $10 \%$ do consumo do dia anterior foi fornecida. As fêmeas foram ordenhadas manualmente, duas vezes ao dia (às $6 \mathrm{~h} 30 \mathrm{e}$ às $16 \mathrm{~h}$ ).

Os animais foram distribuídos em três grupos, de acordo com o escore da condição corporal (ECC) ao parto (MorandFehr \& Hervieur, 1999), em escala de 1 a 5, com intervalos de 0,25 ponto: baixo (ECC entre 1,00 e 2,75), 26 animais; intermediário (ECC acima de 2,75 até o valor 3,50), 27 animais; e alto (ECC acima de 3,50 até 5,00), 15 animais.

As avaliações e coletas de amostras foram iniciadas imediatamente após o parto até a oitava semana de lactação. Durante esse período experimental, foi avaliado o consumo voluntário diário dos animais, por meio da pesagem diária da dieta fornecida e da sobra do dia seguinte. Amostras da dieta e das sobras de cada animal foram coletadas três vezes por semana, para preparação de uma amostra composta semanal, que foi submetida à análise bromatológica para determinação dos teores de matéria seca (MS), cinzas, proteína bruta (PB), extrato etéreo (EE) e lignina, segundo metodologia descrita por Silva (1990), e fibra em detergente neutro (FDN) e fibra em detergente ácido (FDA), segundo Van Soest et al. (1991).

Foi realizada pesagem semanal dos animais utilizando-se balança digital, antes do fornecimento da dieta da manhã, com a primeira pesagem realizada imediatamente após o parto. O escore da condição corporal (ECC) dos animais foi avaliado semanalmente pelo método subjetivo de observação tátil da região dorso-lombar, para estimar o depósito de tecido gorduroso e massa muscular, segundo

Tabela 1 - Composição da ração experimental

\begin{tabular}{lc}
\hline Ingrediente & \% MS da dieta \\
\hline Silagem de milho & 52,94 \\
Fubá de milho & 27,25 \\
Farelo de trigo & 11,03 \\
Farelo de glúten de milho & 5,00 \\
Ureia & 1,20 \\
Minerais & 2,58 \\
Composição bromatológica & \\
Proteína bruta (\%) & 18,00 \\
Proteína degradável no rúmen (\%) & 11,50 \\
Proteína não-degradável no rúmen (\%) & 6,85 \\
Fibra em detergente neutro (\%) & 31,46 \\
Fibra em detergente ácido (\%) & 17,57 \\
Extrato etéreo (\%) & 2,60 \\
Carbohidratos não-fibrosos (\%) & 38,00 \\
Lignina (\%) & 1,27 \\
Cinzas (\%) & 1,18 \\
Cálcio & 0,80 \\
Fósforo & 0,30 \\
Energia líquida (Mcal/kg de MS) ${ }^{1}$ & 1,70 \\
\hline
\end{tabular}

${ }^{1}$ EL estimada de acordo com Moe \& Tyrrell (1975) EL = 0,0245*NDT-0,12 
Morand-Fehr \& Hervieur (1999), com a primeira avaliação realizada imediatamente após o parto.

A produção leiteira dos animais foi avaliada pela pesagem diária e individual do leite. Amostras semanais de leite foram coletadas de cada animal pela manhã e à tarde e enviadas para o Centro Nacional de Produção de Gado de Leite (Embrapa/CNPGL) para avaliação dos constituintes do leite (gordura, proteína, lactose e extrato seco total), por espectrofotometria de infravermelho.

Os resultados de produção leiteira foram corrigidos para 3,5\% de gordura por meio da fórmula demonstrada a seguir (Adams et al., 1995).

PLC 3,5\% $=(0,4255 \times \mathrm{PL})+[16,425 \times(\% \mathrm{G}, 100) \times \mathrm{PL}]$ em que: PLC 3,5\% = produção de leite corrigida para 3,5\% de gordura; $\mathrm{PL}=$ produção de leite $(\mathrm{g} / \mathrm{dia}) ; \mathrm{KG}=$ teor de gordura do leite

O consumo de energia foi estimado com base no cálculo do NDT, segundo Van Soest (1994), transformado em energia líquida, segundo Moe \& Tyrell (1975).

Utilizou-se um delineamento experimental inteiramente casualizado, com três grupos (escores de condição corporal ao parto), cada um com três repetições. Para análise dos dados, foi utilizada análise de variância e as médias comparadas pelo teste de Student Newman-Keuls (SNK), a $5 \%$ de probabilidade. Para o consumo de matéria seca, foi utilizado o procedimento denominado Linear Response Plateau para ajustar modelos de regressão descontínua, partindo de uma regressão linear simples.

\section{Resultados e Discussão}

O escore de condição corporal ao parto não alterou $(\mathrm{P}>0,05)$ os consumos de matéria seca (MS), fibra em detergente neutro (FDN) e proteína bruta (PB), expressos em kg/dia, dos animais, como também o de celulose, expresso em $\mathrm{kcal} / \mathrm{dia}$ e em $\mathrm{kcal} / \mathrm{kg}^{0,75}$. Contudo, quando calculados em \%PV e em $\mathrm{g} / \mathrm{kg}^{0,75}$, os consumos de MS, FDN e PB diferiram entre os grupos $(\mathrm{P}<0,05)$ e foram menores nas cabras gordas.

O consumo voluntário de matéria seca é correlacionado negativamente com as reservas corporais no pósparto em muitos estudos na espécie bovina (Holter et al., 1990; Emery, 1993; Santos, 1996), na espécie ovina (Ingvartsen \& Andersen, 2000) e na espécie caprina (Rodrigues et al., 2007). Essa correlação negativa pode ser justificada pela utilização mais rápida de substratos lipogênicos pelas fêmeas magras, em relação às fêmeas gordas, com menor acúmulo de precursores lipogênicos no sangue, estimulando assim o maior consumo de alimento nas fêmeas magras. Essas observações suportam a teoria glicostática da regulação do apetite proposta por Kennedy, em 1953, de que existe uma relação entre a concentração de glicose plasmática e a regulação do apetite (Guyton, 1992).

Rodrigues et al. (2007), em pesquisa com cabras no período de transição, verificaram efeito do ECC no consumo de MS e FDN no período pré-parto, de modo que os animais com ECC acima de 3,25 apresentaram menor consumo em comparação às cabras com ECC abaixo de 3,25 . Esse resultado justifica que as cabras com ECC acima de 3,25, por possuírem maior quantidade de reservas de energia, tiveram suas necessidades nutricionais supridas com menor quantidade de alimentos, enquanto as cabras com ECC inferior a 3,25 consumiram mais alimento na tentativa de suprir as necessidades nutricionais dessa fase de produção, além de repor ou aumentar alguma reserva de energia.

Esses autores não observaram efeito do ECC sobre o consumo de MS, energia líquida e FDN no período pósparto. Apesar de o ECC não ter afetado diretamente o consumo de MS e celulsose, as cabras com menor ECC consumiram maiores quantidades de FDN, sugerindo que aqueles animais utilizaram mecanismos para aumentar o consumo, uma vez que se apresentaram em condição fisiológica menos adequada que a dos animais com maior

Tabela 2 - Consumo de nutrientes em cabras Alpinas no início da lactação

\begin{tabular}{lcc}
\hline & & Escore de condição corporal ao parto \\
\cline { 2 - 3 } & \multicolumn{1}{c}{ Baixo } & Intermediário \\
\hline Matéria seca (kg/dia) & $1,96 \pm 0,15$ & $1,97 \pm 0,16$ \\
Matéria seca (\%PV) & $3,55 \pm 0,27 \mathrm{a}$ & $3,29 \pm 0,28 \mathrm{a}$ \\
Matéria seca (g/kg,75) & $96,5 \pm 0,75 \mathrm{a}$ & $91,3 \pm 0,75 \mathrm{a}$ \\
Proteína bruta (kg/dia) & $0,37 \pm 0,01$ & $0,37 \pm 0,01$ \\
Proteína bruta (\%PV) & $0,67 \pm 0,02 \mathrm{a}$ & $0,62 \pm 0,03 \mathrm{a}$ \\
Fibra em detergente neutro (kg/dia) & $0,69 \pm 0,06$ & $0,70 \pm 0,07$ \\
Fibra em detergente neutro (\%PV) & $1,25 \pm 0,11 \mathrm{a}$ & $0,35 \pm 0,03$ \\
Celulose (Mcal/dia) & $3.530 \pm 260$ & $0,52 \pm 0,04 \mathrm{~b}$ \\
Celulose (kcal/kg0,75) & $160 \pm 10$ & $0,11 \mathrm{~b}$ \\
\hline
\end{tabular}

Médias com diferentes letras na mesma linha diferem entre si pelo teste SNK $(\mathrm{P}<0,05)$.

CCB = cabras com ECC entre 1,0 e 2,75; CCI = cabras com ECC entre 2,75 até 3,5; CCA = cabras com ECC superior a 3,5 até 5,0. 
ECC. Essas observações sugerem que, quanto mais magras, maior a utilização de mecanismos para redução do déficit energético corporal, similar ao comportamento de ganho compensatório observado na fase de crescimento de animais depois de um período de deficiência nutricional.

Cabras gordas apresentaram menor consumo de FDN, sugerindo maior seleção da dieta para satisfazer rapidamente suas necessidades energéticas, ingerindo menores quantidades de fibra, o que seria importante em animais gordos pela maior limitação de espaço intra-abdominal.

Com base no consumo médio de FDN (\% PV) pelos animais nas três condições corporais $(1,25 ; 1,16$ e 0,94 , respectivamente), sugere-se que o controle fisiológico da regulação de consumo foi o predominante, com as cabras regulando o consumo para satisfazer suas exigências de energia. Essa afirmação apoia-se nos achados de Carvalho et al. (2006), em que o consumo FDN de cabras, com 1,4 a 1,9\% PV de peso passaria a limitar o consumo pela restrição física do trato digestivo de suportar maior quantidade de alimento.

Durante os primeiros 60 dias de lactação, o consumo de MS teve comportamento semelhante entre os animais nas três condições, com crescimento do consumo nas primeiras semanas pós-parto, quando ocorre estabilização ou desaceleração do consumo de MS. Esse comportamento do consumo voluntário foi observado em animais no pós-parto em outros estudos com caprinos (Rodrigues et al., 2007), assim como na espécie bovina (Grummer, 1995).

De acordo com a análise de regressão - Linear Response Plateau (LRP) (Figura 1), o consumo dos animais dos três grupos foi recuperado aos 16 dias (2,3 semanas) pós-parto, com estabilização até o final do período experimental.

Em vacas leiteiras, a redução gradual no consumo de matéria seca inicia-se cerca de três semanas no pré-parto, e pode chegar até 30 a 35\%. Essa diminuição do consumo voluntário permanece no pós-parto, principalmente nas duas primeiras semanas, e aumenta gradativamente até a estabilização. A maior inibição ocorre justamente quando aumenta a demanda por nutrientes para suportar o crescimento fetal e o início da lactação (Bertics et al., 1992; Grummer, 1995). Essas alterações no consumo também foram observadas em cabras no período de transição (Rodrigues et al., 2007).

Houve pequena variação na perda de peso nas primeiras semanas de lactação (Tabela 3) e essa perda foi mais pronunciada na primeira semana em todas as condições corporais, com recuperação iniciando a partir da segunda e terceira semana de lactação.

Houve efeito do ECC ao parto $(\mathrm{P}<0,05)$ sobre o peso corporal dos animais durante quase todo o período
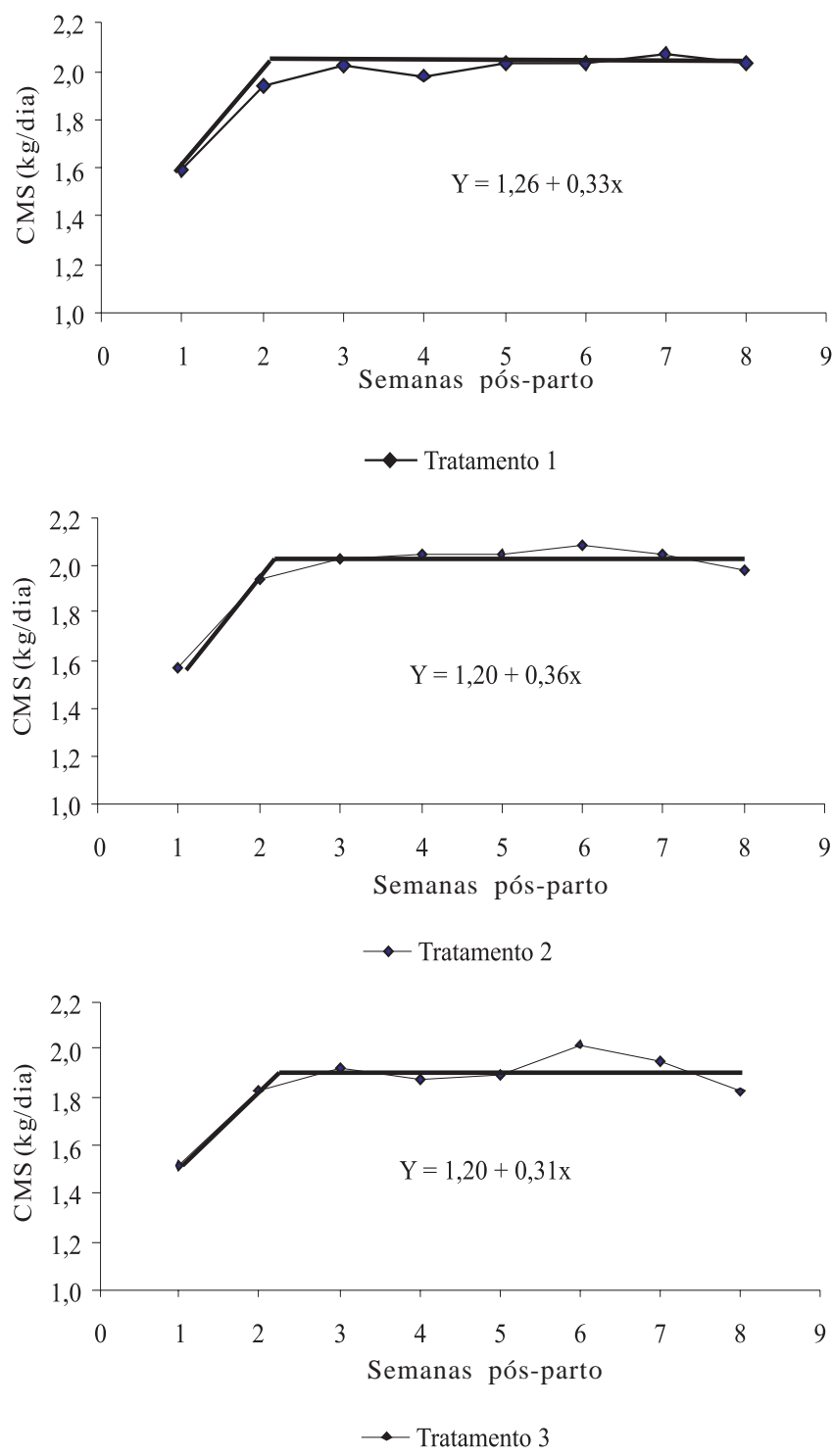

Figura 1 - Consumo de matéria seca dos animais magros (A), com condição corporal intermediária (B) e gordos (C) no período pós-parto.

experimental. Os animais com escore de condição corporal baixo e intermediário não diferiram entre si na sétima e oitava semanas de lactação, o que sugere condições semelhantes para esses animais a partir deste período, provavelmente em decorrência do aumento no consumo de MS nas cabras mais magras durante a fase inicial da lactação, embora não tenha sido observada diferença entre cabras magras e com condição corporal intermediária.

Os animais de condição corporal alta começaram a ganhar peso mais precocemente, a partir da segunda semana, em comparação aos dos outros grupos, nos quais o ganho de peso ocorreu apenas na terceira semana. A média de perda de peso nas duas primeiras semanas foi de $1,20 \mathrm{~kg}$ no grupo de condição corporal alta e de $0,80 \mathrm{~kg}$ e 0,57 kg para 
Tabela 3 - Peso corporal dos animais dos três grupos do parto até a oitava semana do pós-parto

\begin{tabular}{cccr}
\hline Semanas pós-parto & \multicolumn{3}{c}{ Peso vivo $(\mathrm{kg})$} \\
\cline { 2 - 4 } & ECC entre 1,0 e 2,75 & ECC entre 2,75 até 3,5 & ECC superior a 3,5 até 5,0 \\
\hline 0 & $57,4 \pm 7,8 \mathrm{c}$ & $61,7 \pm 6,7 \mathrm{~b}$ & $69,4 \pm 9,1 \mathrm{a}$ \\
1 & $55,7 \pm 7,6 \mathrm{c}$ & $60,6 \pm 6,6 \mathrm{~b}$ & $67,1 \pm 7,7 \mathrm{a}$ \\
2 & $55,4 \pm 7,2 \mathrm{c}$ & $60,6 \pm 6,3 \mathrm{~b}$ & $67,0 \pm 7,8 \mathrm{a}$ \\
3 & $55,0 \pm 7,4 \mathrm{c}$ & $60,0 \pm 6,3 \mathrm{~b}$ & $67,4 \pm 7,7 \mathrm{a}$ \\
4 & $55,8 \pm 7,3 \mathrm{c}$ & $60,3 \pm 6,6 \mathrm{~b}$ & $67,6 \pm 8,1 \mathrm{a}$ \\
6 & $56,2 \pm 7,0 \mathrm{c}$ & $60,4 \pm 6,6 \mathrm{~b}$ & $67,5 \pm 8,1 \mathrm{a}$ \\
7 & $56,0 \pm 7,1 \mathrm{c}$ & $60,4 \pm 6,8 \mathrm{~b}$ & $67,8 \pm 8,5 \mathrm{a}$ \\
8 & $56,2 \pm 7,2 \mathrm{~b}$ & $59,9 \pm 6,7 \mathrm{~b}$ & $67,7 \pm 9,2 \mathrm{a}$ \\
\hline
\end{tabular}

Médias com diferentes letras na mesma linha diferem entre si pelo teste SNK $(\mathrm{P}<0,05)$.

aqueles dos grupos com condição corporal baixa e intermediária, respectivamente. Após esse período, os animais de todos os grupos mantiveram peso corporal constante.

Houve perda de peso dos animais em todos os grupos, justificada pela inibição inicial do consumo voluntário nas primeiras semanas pós-parto, além de maior perda de peso entre os animais gordos, que coincidiu com a maior inibição de consumo apresentada por esses animais. Contudo, apesar dessa maior inibição de consumo, esses animais conseguiram recuperá-lo mais rapidamente e, como consequência, houve recuperação do peso.

Os animais apresentaram média de 2,48; 3,28 e 4,00 de ECC ao parto para o grupo de cabras magras, com condição corporal intermediária e gordas, respectivamente (Tabela 4). A diferença de ECC entre os animais dos três grupos permaneceu durante todo o período experimental, comprovando que o peso corporal não se reflete no ECC dos animais. A perda de peso pequena e gradativa dos animais durante o período avaliado não foi suficiente para promover grandes alterações nos valores de ECC; houve apenas pequena variação nos valores de ECC dos animais durante todo o período experimental.

Tem-se observado diminuição do peso corporal de cabras em torno de $6 \mathrm{~kg}$ durante as primeiras 6 a 12 semanas depois do parto. Com base em estudos do metabolismo do tecido adiposo, Chilliard (1985) e Morand-Fehr et al. (1987) sugeriram que cabras perdem em torno de $1 \mathrm{~kg}$ de peso vivo por semana no primeiro mês no pós-parto e $0,5 \mathrm{~kg} / \mathrm{semana} \mathrm{no}$ mês posterior. Assim como em vacas e ovelhas, a extensão da perda de peso em cabras no pós-parto varia amplamente e é afetada por vários fatores, particularmente o nível de consumo de energia pré e pós-parto. Contudo, todas as pesquisas apontam mobilização das reservas corporais de gordura e proteína nos estágios iniciais da lactação de cabras, apesar de não ser necessariamente correspondente à variação de mudança de peso corporal (AFRC, 1997).

Desta forma, a perda de peso observada neste experimento, de 200 a $260 \mathrm{~g} / \mathrm{semana}$, não foi suficiente para causar diferenças nos valores de ECC dos animais. Normalmente há grandes variações de peso e de ECC no início do pós-parto, justamente pela diminuição do consumo voluntário que promove grande mobilização das reservas corporais, diminuindo assim o peso corporal e a condição corporal. Essa perda pode ter sido reduzida pela dieta com altos valores de energia líquida fornecida aos animais. Rodrigues et al. (2007) também não observaram efeito da condição corporal sobre o ganho ou a perda de peso das cabras no período após o parto, fato explicado pela faixa de condição corporal utilizada, entre 2,5 e 4,0, que provavelmente forneceu condição corporal que promoveu reservas suficientes para que as cabras pudessem apresentar desempenho satisfatório, sem grandes perdas de peso corporal.

Tabela 4 - Variações no escore da condição corporal (ECC) e peso corporal de cabras no pós-parto

\begin{tabular}{lrrr}
\hline & & Escore de condição corporal & \multicolumn{1}{c}{ Alto } \\
\cline { 2 - 4 } Parâmetro & Baixo & Intermediário & $69,40 \pm 9,10$ \\
\hline Peso ao parto $(\mathrm{kg})$ & $57,40 \pm 7,80$ & $61,70 \pm 6,70$ & $68,00 \pm 9,40$ \\
Peso final ${ }^{1}$ & $55,80 \pm 7,20$ & $59,70 \pm 6,90$ & $-1,46 \pm 4,73$ \\
Perda de peso $(\mathrm{kg})^{2}$ & $-1,64 \pm 4,06$ & $-2,02 \pm 3,65$ & $4,00 \pm 0,20$ \\
ECC ao parto & $2,48 \pm 0,30$ & $3,28 \pm 0,20$ & $3,67 \pm 0,50$ \\
ECC final $^{1}$ & $2,54 \pm 0,50$ & $3,00 \pm 0,40$ & $-0,33 \pm 0,44$ \\
Perda de ECC $^{2}$ & $0,06 \pm 0,47$ & $-0,28 \pm 0,40$ &, \pm 40 \\
\hline
\end{tabular}

${ }^{1}$ Observação na oitava semana.

2 Diferença entre os valores observados entre o parto e a última semana. 
Alguns autores relatam que a condição corporal influencia o ganho ou perda de peso dos animais e que a recuperação das perdas ocorre mais tardiamente nos animais de baixa condição corporal ao parto (Garnsworthy \& Topps, 1982). Honhold et al. (1989), utilizando o método de avaliação do escore da condição corporal em caprinos, mencionaram que mudanças na condição corporal foram correlacionadas a mudanças de peso corporal $(\mathrm{r}=0,5, \mathrm{P}<0,01)$ para todas as idades, de modo que a mudança do escore da condição corporal de uma unidade representou mudança média de $12 \%$ no peso corporal.
A média geral das oito semanas no pós-parto e de cada semana individualmente da produção de leite e de seus constituintes não diferiu entre os animais dos grupos ( $\mathrm{P}>0,05)$, diferindo apenas a produção de gordura dos animais de alta condição corporal nas duas primeiras semanas de lactação $(\mathrm{P}<0,05)$. Da mesma forma, Ruegg \& Milton (1995) e Rodrigues et al. (2006), em pesquisa com com caprinos, e Santos (1996) e Lago et al. (2001), trabalhando com bovinos de leite, também não encontraram efeito do ECC ao parto no desempenho produtivo dos animais e nos constituintes do leite (Tabelas 5 e 6).

Tabela 5 - Produção de leite e produção corrigida para 3,5\% de gordura dos animais de três condições corporais ao parte durante as oito primeiras semanas no pós-parto

\begin{tabular}{|c|c|c|c|c|c|c|}
\hline \multirow[t]{2}{*}{ Semanas pós-parto } & \multicolumn{3}{|c|}{ Produção de leite (kg/dia) } & \multicolumn{3}{|c|}{ Produção corrigida para 3,5\% de gordura } \\
\hline & ECC baixo & ECC intermediário & ECC alto & ECC baixo & ECC intermediário & ECC alto \\
\hline 1 & $2,3 \pm 0,8$ & $2,4 \pm 0,8$ & $1,9 \pm 0,6$ & $3,1 \pm 1,2$ & $3,1 \pm 1,2$ & $2,3 \pm 0,7$ \\
\hline 2 & $3,0 \pm 0,7$ & $3,0 \pm 0,9$ & $2,5 \pm 0,6$ & $3,7 \pm 1,0$ & $3,7 \pm 1,2$ & $2,8 \pm 0,7$ \\
\hline 3 & $3,1 \pm 0,8$ & $3,1 \pm 0,9$ & $2,6 \pm 0,6$ & $3,6 \pm 1,1$ & $3,6 \pm 1,1$ & $2,8 \pm 0,7$ \\
\hline 4 & $3,1 \pm 0,9$ & $3,2 \pm 0,9$ & $2,6 \pm 0,7$ & $3,5 \pm 1,0$ & $3,6 \pm 1,2$ & $2,7 \pm 0,8$ \\
\hline 5 & $3,1 \pm 0,9$ & $3,2 \pm 0,9$ & $2,6 \pm 0,7$ & $3,4 \pm 1,0$ & $3,4 \pm 1,1$ & $2,6 \pm 0,7$ \\
\hline 6 & $3,1 \pm 0,9$ & $3,1 \pm 0,9$ & $2,6 \pm 0,7$ & $3,3 \pm 1,0$ & $3,5 \pm 1,0$ & $2,7 \pm 0,6$ \\
\hline 7 & $3,0 \pm 0,9$ & $3,0 \pm 0,9$ & $2,6 \pm 0,7$ & $3,2 \pm 0,9$ & $3,2 \pm 1,0$ & $2,7 \pm 0,6$ \\
\hline 8 & $2,9 \pm 0,9$ & $2,9 \pm 0,9$ & $2,6 \pm 0,8$ & $3,1 \pm 1,0$ & $3,1 \pm 1,0$ & $2,6 \pm 0,7$ \\
\hline Médias & $3,0 \pm 0,2$ & $3,0 \pm 0,2$ & $2,5 \pm 0,2$ & $3,4 \pm 0,2$ & $3,4 \pm 0,2$ & $2,6 \pm 0,1$ \\
\hline
\end{tabular}

CCB = cabras com ECC entre 1,0 e 2,75; CCI = cabras com ECC entre 2,75 até 3,5; CCA = cabras com ECC superior a 3,5 até 5,0.

$\mathrm{PL}=$ produção de leite $(\mathrm{kg} / \mathrm{dia}) ; \mathrm{PLC} 3,5 \%$ = produção de leite corrigida para 3,5\% de gordura.

Não há diferença significativa entre médias pelo teste SNK $(\mathrm{P}>0,05)$.

Tabela 6 - Composição do leite de cabras de três condições corporais ao parto durante as oito primeiras semanas no pós-parto

\begin{tabular}{|c|c|c|c|c|c|c|c|c|c|}
\hline \multirow[t]{2}{*}{ Semanas pós-parto } & \multicolumn{3}{|c|}{ Gordura (\%) } & \multicolumn{3}{|c|}{ Lactose (\%) } & \multicolumn{3}{|c|}{ Proteína (\%) } \\
\hline & $\begin{array}{c}\text { ECC } \\
\text { baixo }\end{array}$ & $\begin{array}{c}\text { ECC } \\
\text { intermediário }\end{array}$ & $\begin{array}{l}\text { ECC } \\
\text { alto }\end{array}$ & $\begin{array}{c}\text { ECC } \\
\text { baixo }\end{array}$ & $\begin{array}{c}\text { ECC } \\
\text { intermediário }\end{array}$ & $\begin{array}{l}\text { ECC } \\
\text { alto }\end{array}$ & $\begin{array}{c}\text { ECC } \\
\text { baixo }\end{array}$ & $\begin{array}{c}\text { ECC } \\
\text { intermediário }\end{array}$ & $\begin{array}{l}\text { ECC } \\
\text { alto }\end{array}$ \\
\hline 1 & $5,5 a$ & $5,3 a$ & $3,8 b$ & 4,6 & 4,7 & 4,6 & 3,7 & 3,5 & 3,7 \\
\hline 2 & $4,7 \mathrm{a}$ & $4,9 a$ & $4,2 b$ & 4,6 & 4,6 & 4,7 & 3,1 & 3,3 & 3,2 \\
\hline 3 & 4,4 & 4,4 & 3,9 & 4,7 & 4,8 & 4,8 & 2,9 & 2,9 & 2,9 \\
\hline 4 & 4,2 & 4,3 & 3,8 & 4,8 & 4,8 & 4,8 & 2,7 & 2,7 & 2,8 \\
\hline 5 & 4,0 & 3,9 & 3,7 & 4,8 & 4,6 & 4,7 & 2,8 & 2,9 & 2,8 \\
\hline 6 & 4,0 & 4,2 & 3,7 & 4,7 & 4,7 & 4,8 & 2,8 & 2,9 & 2,9 \\
\hline 7 & 3,8 & 3,9 & 3,7 & 4,7 & 4,6 & 4,7 & 2,7 & 2,8 & 2,9 \\
\hline 8 & 3,9 & 3,7 & 3,5 & 4,7 & 4,6 & 4,8 & 2,8 & 2,9 & 3,0 \\
\hline Médias & $4,3 \pm 0,5$ & $4,3 \pm 0,5$ & $3,9 \pm 0,4$ & $4,7 \pm 0,1$ & $4,7 \pm 0,1$ & $4,7 \pm 0,1$ & $3,0 \pm 0,3$ & $3,0 \pm 0,2 \quad 3$ & $3,0 \pm 0,2$ \\
\hline
\end{tabular}

Médias com diferentes letras na mesma linha diferem entre si pelo teste SNK $(\mathrm{P}<0,05)$.

CCB $=$ cabras com ECC entre 1,0 e 2,75; CCI = cabras com ECC entre 2,75 até 3,5; CCA = cabras com ECC superior a 3,5 até 5,0.

Os valores médios de lactose e de proteína do leite são similares àqueles encontrados na literatura para a espécie caprina (Rodrigues et al., 2006; Carvalho et al., 2006). Contudo, os valores de gordura foram um pouco acima dos valores médios reportados na literatura para cabras Alpinas e Saanen.

\section{Conclusões}

O melhor consumo voluntário ocorreu até 3,5 de escore da condição corporal. As variações de peso corporal e de escore da condição corporal diminuem em cabras leiteiras de média produção, independentemente da condição 
corporal ao parto, quando fornecida dieta de alta qualidade e com altos níveis de energia.

\section{Agradecimentos}

À Universidade Federal de Viçosa (UFV) e ao Centro Nacional de Pesquisa (CNPq), pelo apoio financeiro na realização deste trabalho.

\section{Literatura Citada}

ADAMS, R.; COMERFORD, J.W.; FORD, S.A. et al. Dairy nutrition. Dairy reference manual. 3.ed. Ithaca: Northeast Regional Agricultural Engineering Service, 1995. p.129.

AGRICULTURAL AND FOOD RESEARCH COUNCIL - AFRC. Technical Committee on Responses to Nutrients. The Nutrition of goats. Nutrition Abstracts and Reviews (Series B), v.67, n.11, 1997. (Report, 10).

BERTICS, S.J.; GRUMMER, R.R.; CADORNIGA-VALINO, C. et al. Effect of prepartum dry matter intake on liver triglyceride concentration in early lactation. Journal of Dairy Science, v.75, p.1914, 1992.

CARVALHO, S.; RODRIGUES, M.T.; BRANCO, R.H. et al. Consumo de nutrientes, produção e composição do leite de cabras da raça Alpina alimentadas com dietas contendo diferentes teores de fibra. Revista Brasileira de Zootecnia, v.35, n.3, p.1154-1161, 2006 (supl.).

EMERY, R.S. Energy needs of dry cows. In: TRI-STATE DAIRY NUTRITION CONFERENCE, 1993, Fort Wayne. Proceedings... Fort Wayne, 1993. 35p.

GARNSWORTHY, P.C.; TOPPS, J.H. The effect of body condition of dairy cows at calving on their food intake and performance when given complete diets. Animal Production, v.35, n.1, p.113-119, 1982.

GRUMMER, R.R. Impact of changes in organic nutrients metabolism on feeding the transition cow. Journal of Animal Science, v.73, p.2820-2833, 1995.

GUYTON, C.A. Tratado de fisiologia médica. 2.ed. Rio de Janeiro: Guanabara Koogan, 1992. 846p.

HOLTER, J.B.; SLOTNICK, M.J.; HAYES, H.H. et al. Effect of prepartum dietary energy on condition score, postpartum energy, nitrogen partition, and lactation production responses. Journal of Dairy Science, v.73, p.3502-1511, 1990.
HONHOLD, N.; PETIT, H.; HALLIWELL, R.W. Condition scoring scheme for small east African goats in Zimbabwe. Tropical Animal Health Production, v.21, n.2, p.121-127, 1989.

INGVARTSEN, K.L.; ANDERSEN, J.B. Integration of metabolism and intake regulation: a review focusing on periparturient animals. Journal of Dairy Science, v.83, p.1573-1597, 2000.

LAGO, E.P.; PIRES, A.V.; SUSIN, I. et al. Efeito da condição corporal ao parto sobre alguns parâmetros do metabolismo energético, produção de leite e incidência de doenças no pósparto de vacas leiteiras. Revista Brasileira de Zootecnia, v.30, n.5, p.1544-1549, 2001.

MOE, P.W.; TYRRELL, H. F. Symposium: production efficiency in the high producing cow. Efficiency of conversion of digested energy to milk. Journal of Dairy Science, v.58, p.602-610, 1975.

MORAND-FEHR, P.; HERVIEU, J. Apprécier l'éat corporel des chèvres: Intérêt et méthod. Reussir La Chevre, n.231, p.22-34, 1999.

RENNÓ, F.P; PEREIRA, J.C.; SANTOS, A.D.F. et al. Efeito da condição corporal ao parto sobre a produção e composição do leite, curva de lactação e mobilização de reservas corporais em vacas Holandesas primíparas e multíparas. Arquivo Brasileiro de Medicina Veterinária e Zootecnia, v.58, n.2, p.220-233, 2006

RODRIGUES, C.A.F.; RODRIGUES, M.T.; BRANCO, R.H. et al. Avaliação do consumo e de metabólitos plasmáticos de cabras gestantes, com duas condições corporais, alimentadas com dietas apresentando diferentes níveis de energia. Revista Brasileira de Zootecnia, v.36, p.945-952, 2007.

RODRIGUES, C.A.F.; RODRIGUES, M.T.; BRANCO, R.H. et al. Influência da condição corporal e da concentração de energia nas dietas no periparto sobre a performance de cabras em lactação. Revista Brasileira de Zootecnia, v.34, p.1560-1567, 2006.

RUEGG, P.L.; MILTON, R.L. Body condition score of holstein cow on Prince Edward Island, Canada: Relationships with yield, reproductive performance, and disease. Journal of Dairy Science, v.78, p.552-564, 1995.

SANTOS, J.E.P. Effect of degree of fatness prepartum on lactational performance and follicular development of early lactating dairy cows. 1996. 107f. Thesis (Master of Animal Science) - University of Arizona, Arizona, 1996.

SILVA, D.J. Análise de alimentos (Métodos químicos e biológicos) Viçosa, MG: Editora UFV, 1990. 166p.

VAN SOEST, P.J. Integrated feeding systems. In: Nutritional ecology of the ruminant. 2.ed. Ithaca, USA: Cornell University Press, 1994. 476p.

VAN SOEST, P.J.; ROBERTSON, J.B.; LEWIS, B.A. Symposium: carbohydrate methodology, metabolism, and nutritional implications in dairy cattle. Journal of Dairy Science, v.74, n.10, p.3583-3597, 1991. 